the associations and superassociations. How did these bodies ever get confined to this plane? If we imagine stars being formed from the interstellar medium, the location of associations in the plane is obvious, and the restriction of gas to the plane is understood as the result of the collapse of the primordial cloud. However, we know no mechanism which would have brought the (primordial) superdense bodies into the plane; for then we would rather expect to find a distribution like that of the halo stars. What do you think about this?

Ambartsumian: Of course we don't know the forces which can bring the superdense prestellar formations into the galactic plane, but it is possible that some mechanism exists.

Feast: It may be of interest to remark that quite apart from size there may be other differences between superassociations like 30 Doradus and normal associations. For instance, 30 Doradus seems to contain a much higher proportion of WR stars than the associations so far studied in the Galaxy.

de Vaucouleurs: You have indicated that 30 Doradus is a superassociation, but that NGC 604 does not qualify. Could you describe more precisely what the operational definition of superassociation is?

Ambartsumian: We define the superassociations by the absolute brightness ( $M$ brighter than $-13 \cdot 5$ ) and colours. It seems that NGC 604 falls somewhat on the borderline between normal and superassociations.

\title{
30. LARGE HI CLOUDS IN THE GALAXY
}

\author{
R. X. McGeE
}

CSIRO Radiophysics Laboratory, Sydney

\section{Introduction}

van de Hulst (1958), in giving representative sizes of the structural details of the interstellar medium, lists the dimensions of a typical cloud, seen in $21-\mathrm{cm}$ emission, as $20-70$ pc but warns that we should not be surprised to find different "sizes of interstellar clouds" determined from different sets of data.

I wish to introduce two classes of cloud or complex of neutral hydrogen of much larger dimensions than the "typical cloud":

(a) The first type, which has been observed close to the Sun, is an association presumably composed of "typical clouds" each with similar density characteristics and velocity dispersion. In general, a "typical cloud" is hard to distinguish as an individual component.

(b) The second type, observed at greater distances, is a complex several times larger. (Beyond 3 or $4 \mathrm{kpc}$ from the Sun the aerial resolution does not permit such detail as the first type to be delineated.) The clouds occur in succession along the galactic spiral arms and those mentioned here form arms at distances from the galactic centre, $R>R_{0}$ (distance Sun to centre).

The discussion is based on observations made with a low-resolution paraboloid2.2 between half-power points- and the 48-channel $\mathrm{H}$-line receiver. The bandwidth was $38 \mathrm{kc} / \mathrm{s}(\sim 8 \mathrm{~km} / \mathrm{sec}$ in radial velocity) with channel spacing at $33.2 \mathrm{kc} / \mathrm{s}$ $(\equiv 7.0 \mathrm{~km} / \mathrm{sec})$. Profiles were recorded at $2 \mathrm{~min}$ intervals in time at spacings of $1^{\circ}$ in declination between +42 and $-90^{\circ}$. The equipment is described by McGee and Murray (1963). 


\section{Three HI Complexes in the Solar Neighbourhood}

Since the three examples of the first type of complex are rather fully treated in a recent paper by McGee, Murray, and Milton (1963), only a brief account will be given here. The clouds are the well-known southerly bulge in Perseus-TaurusOrion, the spread of HI gas on either side of the galactic plane in Puppis-Vela, and the Scorpius-Ophiuchus complex. Some of the details such as the extent in galactic longitude and latitude, the maximum value over the region of $T_{\max }$. (the temperature at an $\mathrm{H}$-line profile peak, used as a measure of the neutral hydrogen intensity), the average radial velocity (at $T_{\max }$ ) over the cloud, the mean profile halfwidth, the estimated distance in parsecs, the estimated linear dimensions in latitude and longitude, the average density, and remarks are given in Table 1.

In the case of Scorpius-Ophiuchus a "typical cloud" surrounding the HII region near $\zeta$ Ophiuchi is readily distinguished and corresponding quantities have been included in the table for comparison.

The local region, then, would seem to be largely made up of three or four complexes of 100-150 pc in diameter. Reasonable dimensions would be $220 \mathrm{pc}$ in $z$ and $500 \mathrm{pc}$ in each direction along the arm. An earlier estimate (McGee and Murray 1961) for the average density near the Sun is $0.5 \mathrm{H}$ atoms $\mathrm{cm}^{-3}$.

\section{Concentrations of $H I$ at Distances $R>R_{0}$}

In an attempt to show how the neutral hydrogen appears in its simple observational form, the representation in $T_{\max }$. (the profile peak temperature) and the radial velocity at $T_{\max }$., appropriate to the single-peaked profiles in the case of the local gas, has been extended to the three- and more-peaked profiles in the galactic plane region. Contour diagrams of $T_{\max }$. follow the variation of the profile peaks from point to point along and across the galactic plane. Companion diagrams of radial velocity were made.

The low-velocity peaks have been incorporated in the diagrams of Paper II (McGee, Murray, and Milton 1963) of the HI sky survey series. It is hoped to publish the information on peaks at other velocities in Paper III of the series. Thirteen pairs of diagrams have been prepared; some extend only $20^{\circ}$ in longitude, others over $100^{\circ}$. The latitude extent is less than $\pm 10^{\circ}$.

A number of concentrations of $\mathrm{HI}$ appear in each diagram. With the aid of the radial velocity contours and the velocity-distance relation according to a particular model (see, for example, Kerr (1962), Figs. 7 and 8), it is possible to assign positions and distances to these concentrations and, indeed, to the corresponding $T_{\max }$. pattern as a whole.

Thus the 13 contour diagrams are indicators of spiral arms of hydrogen gas or spurs and portions thereof. In most cases the information refers to gas at distances $R<R_{0}$ where an ambiguity of position on the velocity-distance diagram exists. However, in four cases, the large concentrations making up these spiral arms are positioned at distances $R>R_{0}$ and are listed here as members of the second class of complex. The distance from the Sun varies from 3 to $19 \mathrm{kpc}$ to make the aerial resolution equivalent to a range of 115 to $730 \mathrm{pc}$. 


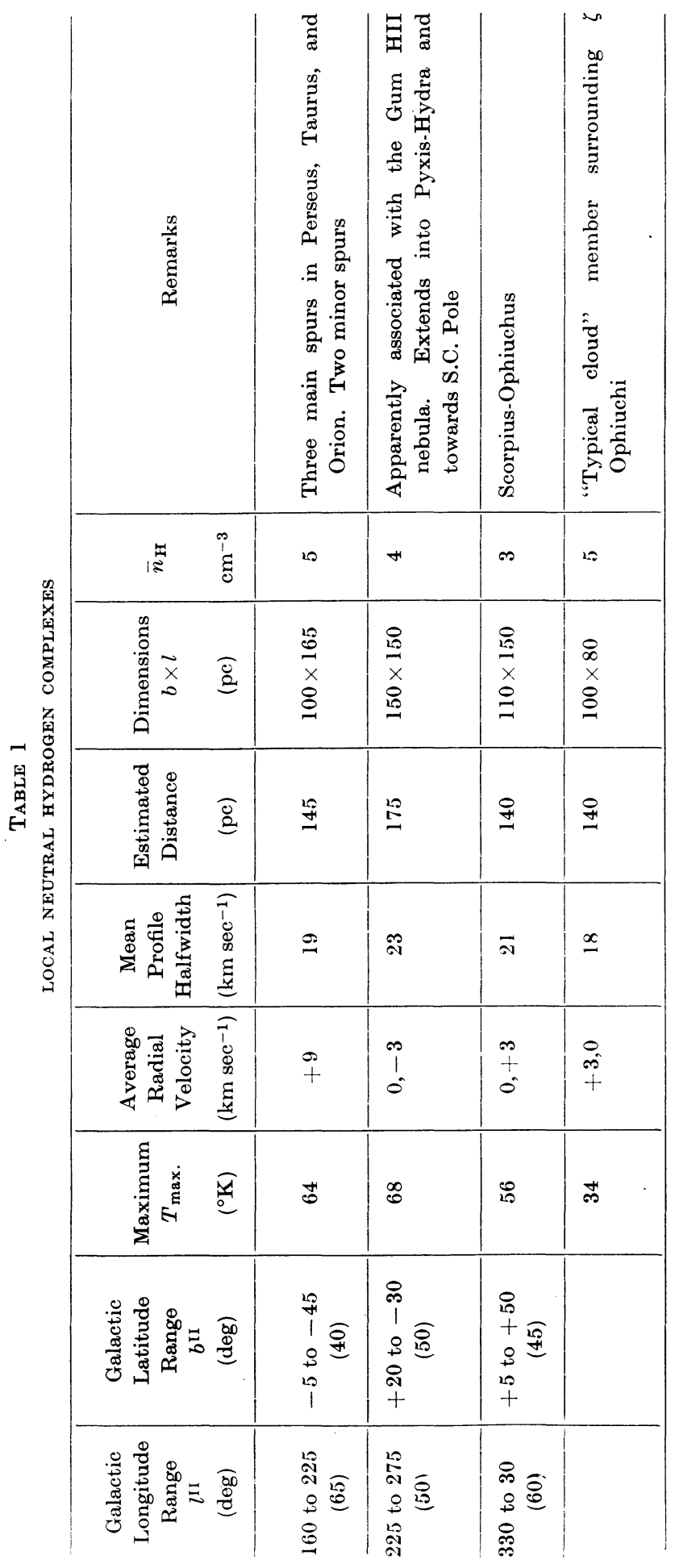


Twenty-seven complexes have been counted but to simplify presentation linear dimensions, densities, and masses of an average complex in each arm have been calculated. The longitude range, the radial velocity range, the approximate distances from the galactic centre for the four arms, and the number of clouds in each arm are also given in Table 2. The upper and lower limits of latitude dimensions are included in column 5 of the table.

The results suggest a class of HI clouds very much larger than any previously mentioned in the literature. Their linear dimensions increase and the densities decrease

TABLE 2

FOUR SPIRAL ARMS OF HI AT DISTANCES $R>R_{0}$

\begin{tabular}{|c|c|c|c|c|c|c|c|}
\hline \multirow{3}{*}{$\begin{array}{c}\text { Galactic } \\
\text { Longitude } \\
\text { Range } \\
l^{\mathrm{II}}\end{array}$} & \multirow{3}{*}{$\begin{array}{c}\text { Radial Velocity } \\
\text { Range } \\
\left(\mathrm{km} \mathrm{sec}^{-1}\right)\end{array}$} & \multirow{3}{*}{$\begin{array}{l}\text { Approx. } R \\
(\mathrm{kpc})\end{array}$} & \multirow{3}{*}{$\begin{array}{l}\text { No. of } \\
\text { Concen- } \\
\text { trations }\end{array}$} & \multicolumn{4}{|c|}{ Average Complex in Spiral Arm } \\
\hline & & & & \multicolumn{2}{|c|}{ Dimensions } & \multirow{2}{*}{$\begin{array}{c}\text { Density } \\
n_{\mathrm{H}} \\
\mathrm{cm}^{-3}\end{array}$} & \multirow{2}{*}{$\begin{array}{c}\text { Mass } \\
M_{\odot}\end{array}$} \\
\hline & & & & $b(\mathrm{pc})$ & $l(\mathrm{pc})$ & & \\
\hline $\begin{array}{c}280-352^{\circ} \\
\left(72^{\circ}\right)\end{array}$ & $+42,+18,+54,+27$ & 9 & 6 & $\begin{array}{c}(420) \\
565 \\
(755)\end{array}$ & 1950 & $0 \cdot 9$ & $10^{7}$ \\
\hline $\begin{array}{c}200-295^{\circ} \\
\left(95^{\circ}\right)\end{array}$ & $+33,+87$ & $10 \cdot 5$ & 4 & $\begin{array}{c}(350) \\
500 \\
(590)\end{array}$ & 1200 & $0 \cdot 9$ & $0.5 \times 10^{7}$ \\
\hline $\begin{array}{r}220-332^{\circ} \\
\left(112^{\circ}\right)\end{array}$ & $+60,+108,+63$ & $12 \cdot 5$ & 11 & $\begin{array}{c}(555) \\
945 \\
(1330)\end{array}$ & 1850 & $0 \cdot 3$ & $10^{7}$ \\
\hline $\begin{array}{r}16-86^{\circ} \\
\left(70^{\circ}\right)\end{array}$ & $-18,-72$ & 12 & 6 & $\begin{array}{c}(1070) \\
1190 \\
(1330)\end{array}$ & 2500 & $0 \cdot 3$ & $2 \times 10^{7}$ \\
\hline
\end{tabular}

with distance from the galactic centre. The picture of a very flat layer of hydrogen of $z=220 \mathrm{pc}$ inside $R=R_{0}$ is supplemented by one of a rapid increase in layer thickness outside $R_{0}$.

At $R=R_{0}, z=220$ pc is consistent with the findings in Section II;

at $R=9-11 \mathrm{kpc}, z=500-600 \mathrm{pc}$;

at $R=12-13 \mathrm{kpc}, z=950-1200$ pc from Table 2 .

The deviation of some hundreds of parsecs from the galactic plane, reported by Kerr, Hindman, and Carpenter (1957), is present in addition.

An important qualification should be noted: in the region $R>R_{0}$ the velocitydistance model is not well known and therefore all the calculations depending on distance are subject to this uncertainty. 


\section{References}

van de Hulst, H. C. (1958).-Rev. Mod. Phys. 30: 913-23. Symp. IAU 8: 913-23. [Cambridge, Mass. 1957.]

KERR, F. J. (1962).-M.N. $123: 327-45$.

Kerr, F. J., Hindman, J. V., and Carpenter, Martha Stahr (1957).-Nature 180: 677-9.

McGee, R. X., and Murray, J. D. (1961).-Aust. J. Phys. 14: 260-78.

McGee, R. X., and Murray, J. D. (1963).—Proc. I.R.E. (Aust.) 24 : 191-6.

McGee, R. X., Murray, J. D., and Milton, Janice A. (1963).-Aust. J. Phys. $16: 136-70$.

\section{Discussion}

Buscombe: Mrs. Kennedy and I (M.N. 124: 195-200 (1962)) have measured interstellar calcium velocities, for clouds in front of several early southern B stars, which agree closely with Mr. McGee's hydrogen results.

Burke: In a survey of the anticentre region we made several years ago at the Carnegie Institution we found a somewhat smaller velocity dispersion within the cloud associated with the Taurus complex-12 km/sec or slightly less. As for the larger structure, associated with the Perseus arm extending across the anticentre, we find, using simple dynamical behaviour as a criterion, a dimension of about $500 \times 1500 \mathrm{pc}$, a dimension very similar to the other large concentrations you have shown.

Davies: Spectra taken with a $12^{\prime}$ beam and a $3 \mathrm{kc} / \mathrm{s}$ bandwidth at Jodrell Bank show three or four velocity features in various parts of the local system. Have you made a study of systematic motions within your extended clouds?

McGee: Not yet.

de Vaucouleurs: Your maps of HI total brightness show a good general and in places a detailed correlation with maps of obscuration from galaxy counts. In addition to the correlation with the Taurus-Orion dark nebula (first noted at Harvard some years ago by Professor Bok and his group) and with the Ophiuchus dark nebula, your HI contours seem to agree well with the extensive obscured area over the region of the South Celestial Pole. In this connection, I wish to call the attention of the Mount Stromlo astronomers to the importance of procuring homogeneous galaxy counts to a faint limiting magnitude over the south celestial polar cap where only preliminary and heterogeneous Harvard data are available.

\section{A LARGE HIGH VELOCITY CLOUD AT $l^{\mathrm{II}}=41^{\circ}, b^{\mathrm{II}}=-15^{\circ}$}

\section{J. H. OORT \\ Leiden Observatory}

In a study made by Miss Gail Smith at Leiden a cloud was found with a rather exceptional velocity and a large velocity spread. The cloud is elongated, with a ratio of length to breadth of about $3: 1$. Its length is about $10^{\circ}$. The average velocity relative to the local standard of rest is $+90 \mathrm{~km} / \mathrm{sec}$; the width of the profiles is considerable, corresponding to a halfwidth of about $30 \mathrm{~km} / \mathrm{sec}$. The mass can be estimated only if we know the distance. Taking $200 \mathrm{pc}$ as a reasonable estimate of the minimum distance of the cloud the minimum mass is about 200 solar masses. The computed mass varies as the square of the distance.

It is possible that we are witnessing here part of an old supernova shell, or rather the gas swept up by a supernova shell, of the same kind as we find in the Cygnus loop. However, the mass of the exploded shell and its original velocity must have been quite high to explain the observed cloud. 